\title{
Functionalization of silicon step arrays II: Molecular orientation of alkanes and DNA
}

J. N. Crain, A. Kirakosian, and J.-L. Lin

Department of Physics, University of Wisconsin Madison, Madison, Wisconsin 53706

Yuedong Gu, Rahul R. Shah, and Nicholas L. Abbott

Department of Chemical Engineering, University of Wisconsin Madison, Madison, Wisconsin 53713

F. J. Himpsel ${ }^{\mathrm{a})}$

Department of Physics, University of Wisconsin Madison, Madison, Wisconsin 53706

(Received 27 December 2000; accepted for publication 26 June 2001)

\begin{abstract}
Alkanes and DNA are adsorbed via thiol groups on ultrathin gold films that have been templated on stepped $\operatorname{Si}(111) 7 \times 7$ surfaces. The orientation of the adsorbed molecules is determined by polarization-dependent near edge x-ray absorption fine structure spectroscopy from the $\mathrm{C} 1 s$ and $\mathrm{N} 1 s$ core levels. An anisotropy in the polar distribution is found which is consistent with models in which the molecules have a preferred tilt angle with respect to normal but a random azimuthal distribution. In order to obtain maximum coverage and an oriented overlayer it is necessary to deposit more than $3 \mathrm{~nm}$ of $\mathrm{Au}$ (12 monolayers). (C) 2001 American Institute of Physics.
\end{abstract}

[DOI: $10.1063 / 1.1397297]$

\section{INTRODUCTION}

Because silicon can be patterned in many ways, it should be possible to use it as a versatile template for combining organic molecules and biomolecules with silicon electronics. Among the possible applications are molecular electronics ${ }^{1,2}$ and biosensors. A patterned surface is required for connecting molecular switches or for growing or locating biological species (e.g., cells or viruses) on a substrate that exhibits their preferred texture. Selective absorption has been achieved on the micrometer scale, but it becomes challenging to perform on the nanometer-scale.

A widely used approach to functionalize inorganic surfaces is through a gold overlayer which allows for the attachment of biomolecules via thiol groups. The sulfur head group bonds with the gold surface. Extensive work has been done with self-assembled monolayers (SAMs) of thiol-modified alkanes on gold. ${ }^{3-7}$ Recent studies have attached larger molecules such as thiol-modified DNA. ${ }^{8}$ A more sophisticated approach for the attachment of larger molecules involves a building-up process. First, a SAM is formed on the Au surface. The functional end groups of the molecules in the SAM can be chosen (or designed through surface chemistry) to provide site-selective binding to specific biological species, such as proteins, DNA, ${ }^{9}$ antibiotics, ${ }^{10}$ or cells. ${ }^{11}$ Such attachment procedures often involve multiple steps and layers, and enable the attachment of a wide variety of different molecules. Such a wide repertoire of processes makes gold an effective and versatile interface between silicon and biological systems. However, most past studies have been based on polycrystalline gold films with unknown nanometer-scale topography (for exceptions, see Refs. 6, 7, and 12-15).

\footnotetext{
a)Electronic mail: fhimpsel@facstaff.wisc.edu
}

In a companion article ${ }^{16}$ we have developed methods to pattern stepped silicon surfaces by self-assembly and to coat them with a conformal gold layer that preserves step patterns. This method provides a surface roughness of only a monolayer of atoms. By utilizing a specific miscut angle and annealing procedure, regular arrays of steps with spacings ranging from 2 to $80 \mathrm{~nm}$ can be prepared. ${ }^{17,18}$ Gold grown on $\mathrm{Si}(111)$ at a thickness of $3 \mathrm{~nm}$ still preserves the well-defined bunched step nanopattern. ${ }^{16} \mathrm{~A}$ titanium adhesion layer facilitates the smooth growth of the gold.

Here we use such surfaces to directly attach organic and biological molecules. Monolayers of hexadecanethiols and short double-stranded DNA segments are adsorbed from solution and compared to similar monolayers on thicker gold films $(13 \mathrm{~nm})$ on glass. The orientation and coverage of the molecules are determined by polarization-dependent near edge $\mathrm{x}$-ray absorption fine structure spectroscopy (NEXAFS).

\section{EXPERIMENTAL METHOD}

Since the X-ray absorption signal is proportional to the number of atoms, comparison of core level absorption spectra can be used to find the coverages of specific atoms, such as $\mathrm{C}$ or N. X-ray absorption spectra can also be used to fingerprint specific chemical species adsorbed on surfaces by the pattern of their unoccupied valence orbitals. ${ }^{19-21} \mathrm{Al}-$ kanethiols can be characterized by the energies of transitions from the $\mathrm{C} 1 s$ core level to the $\mathrm{C}-\mathrm{C}$ and $\mathrm{C}-\mathrm{H} \sigma^{*}$ orbitals and DNA by the transition from the $\mathrm{N} 1 s$ to the $\pi^{*}$ orbitals in the nucleotide bases (Fig. 1). The polarization dependence of the x-ray spectra can be used to determine the orientation of the molecules adsorbed on the surface. The dipole transition element from a $s$-like core level to a $p$-like orbital is approximately proportional to $\cos ^{2} \theta$, where $\theta$ is the angle between the $p$ orbital and the polarization vector of the inci- 


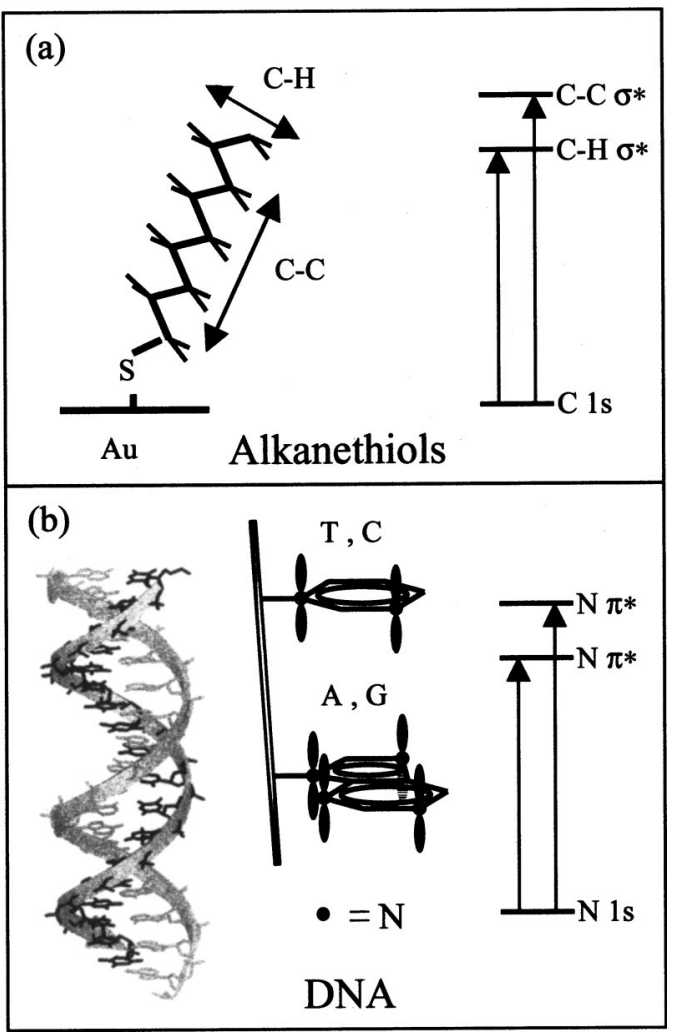

FIG. 1. Determination of the orientation of adsorbed molecules by polarization-dependent absorption spectroscopy from the $\mathrm{C} 1 s$ and $\mathrm{N} 1 s$ core levels. Transitions into antibonding valence orbitals are allowed if the orbital orientation is parallel to the electric field vector of the light. (a) For alkanethiols: The $\mathrm{C}-\mathrm{C}$ orbitals define the axis of the molecule and the $\mathrm{C}-\mathrm{H}$ orbitals the direction perpendicular to the axis and in the plane of the molecule. (b) For DNA: The $\pi^{*}$ orbitals of the nucleotide bases define the axis perpendicular to the purine $(\mathrm{A}, \mathrm{G})$ and pyrimidine $(\mathrm{C}, \mathrm{T})$ rings, which closely follows the axis of the double helix.

dent photons. In the case of molecules, such as alkanethiols and DNA, this bond direction is averaged over all identical bonds within the molecule (Fig. 1). For alkanethiols, if we assume an all-trans conformation, the average $\mathrm{C}-\mathrm{C}$ bond direction defines the axis of the molecule and the average $\mathrm{C}-\mathrm{H}$ bond direction is perpendicular to the axis in the plane of the molecule. For DNA, the $\pi^{*}$ orbitals of the nucleotide bases define the axis perpendicular to the purine $(A, G)$ and pyrimidine $(\mathrm{C}, \mathrm{T})$ rings, which closely follows the axis of the double helix.

NEXAFS was performed at the Synchrotron Radiation Center (SRC). The sample photocurrent was recorded as a measure of the absorption cross section. Absorption spectra were measured at the carbon $1 s$ edge for alkanethiols and at the nitrogen $1 s$ edge for DNA. The signal-to-noise ratio was as low as $5 \times 10^{-4}$ of the total signal.

The preparation of gold-coated Si (111) samples with nano-patterning is described in the companion article. ${ }^{16}$ Atomically flat terraces $(60 \pm 10 \mathrm{~nm})$ separated by facets of 13-15 bunched steps were obtained by a special heating sequence. ${ }^{17}$ Gold was deposited in varying thicknesses $(1.5$, 6 , and 12 monolayers) with a Ti prelayer ( 2 monolayers) for the thicker films (6 and 12 monolayers). As a control, thicker $\mathrm{Au}$ films (13 nm thick) were deposited onto glass using an

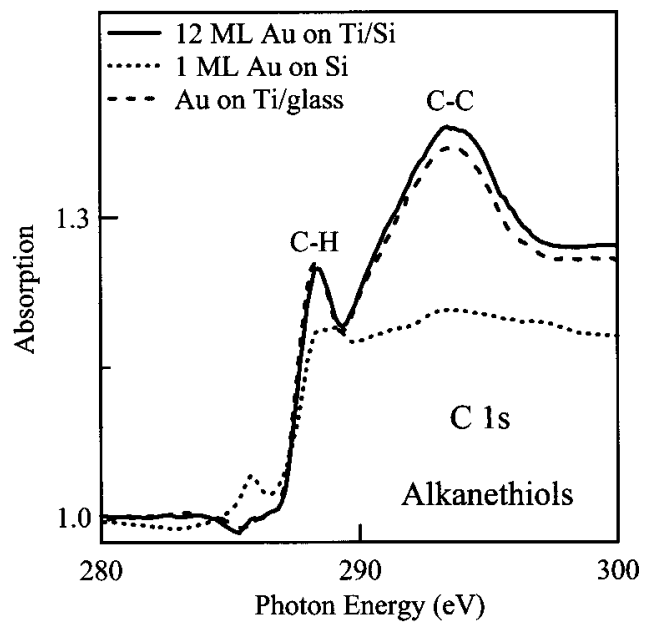

FIG. 2. C $1 s$ absorption edge of a layer of hexadecanethiols adsorbed on silicon and glass surfaces that are functionalized by a gold layer with an intermediate titanium adhesion layer. In order to reach saturation coverage the Au substrate needs to be at least 12 monolayers thick $(3 \mathrm{~nm})$.

electron beam evaporator. A Ti underlayer $(5 \mathrm{~nm})$ facilitated the adhesion of the Au to the glass.

Ethanolic solutions of alkanethiols $(1 \mathrm{mM})$ form a nearly complete self-assembled monolayer on the surface of $\mathrm{Au}$ films in $2 \mathrm{~h}^{3}$ In our work gold films on glass and silicon were immersed in ethanolic solutions of hexadecanethiol (92\%, Aldrich) using concentrations and exposure times ranging from $1 \mathrm{mM}$ for $2 \mathrm{~h}$ to $0.1 \mathrm{mM}$ for $16 \mathrm{~h}$. All samples were thoroughly rinsed with ethanol after removal from solution and were blown dry and transferred directly into vacuum for photoabsorption study.

Thiol-modified double-stranded DNA [5'-HS$\left(\mathrm{CH}_{2}\right)_{6}$ - TG CAG TTC CGG TGG CTG ATC and its reverse complement] was polyacrylamide gel electrophoresis (PAGE) purified after the annealing process to remove unhybridized single-stranded DNA (Alpha DNA). Aqueous solutions of ds-DNA $(1 \mu \mathrm{M})$ were prepared with a TE buffer [10 $\mathrm{mM}$ tris- $\mathrm{Cl}, 1 \mathrm{mM}$ ethylenediaminetetraacetic acid, and $1 \mathrm{M}$ $\mathrm{NaCl}, p \mathrm{H} 7.0]$. Samples were immersed for long $(>24 \mathrm{~h})$ and short $(1 \mathrm{~h})$ durations at room temperature, and the samples were rinsed thoroughly with deionized water.

\section{A. Alkanethiols}

Figure 2 compares the C $1 s$ absorption spectra for monolayers of hexadecanethiols on gold-coated glass and silicon. The raw spectra are normalized to a clean silicon reference signal to remove the transmission function of the optics. The signal at photon energies below the carbon $1 s$ absorption edge is normalized to one in order to account for differences in the absorption depth at different angles. The electron yield is proportional to the electron escape depth over the absorption length, thereby changing with the angle of incidence. It also serves as a rough normalization of the absolute signal, as long as the preedge signal is dominated by the $\mathrm{Au}$ substrate. The $\mathrm{C} 1 s$ absorption is the extra signal above this background. The two primary features are characteristic of alkane monolayers and agree within an experimental error with earlier assignments of transitions from the C $1 s$ 

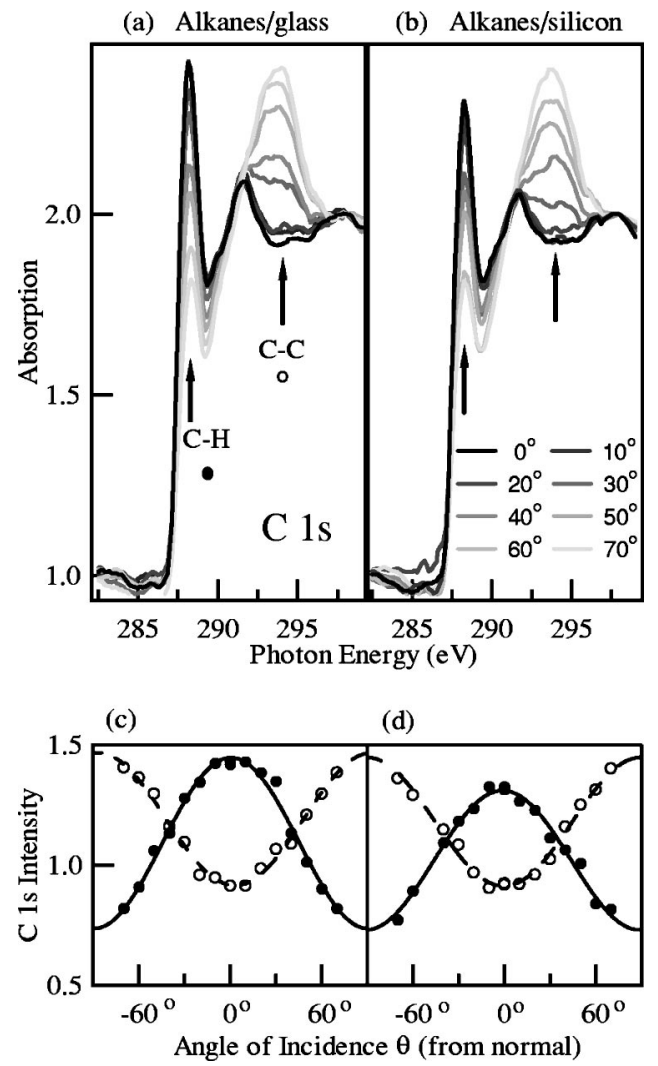

FIG. 3. Polarization dependence of the $\mathrm{C} 1 s$ absorption of hexadecanethiols, measured by varying the angle of incidence $\vartheta$ of $p$-polarized light. The angle $\vartheta$ from the sample normal is equal to the angle of the electric field vector $\mathbf{E}$ from the surface. The $\mathrm{C}-\mathrm{H}$ orbitals are excited preferentially for $\vartheta=0^{\circ}$, the C $-\mathrm{C}$ orbitals for $\vartheta=90^{\circ}$, demonstrating an oriented overlayer. (a) and (c) for a glass substrate, (b) and (d) for stepped silicon.

core level to the unoccupied $(\mathrm{C}-\mathrm{H})$ and $(\mathrm{C}-\mathrm{C}) \sigma^{*}$ orbitals for similar chain length alkanethiols. ${ }^{19}$ Polarization dependent effects suppress the $\mathrm{C}-\mathrm{C}$ resonance at normal incidence. An incidence angle of $60^{\circ}$ was used so that both features are easily distinguishable. The curves for the thick $\mathrm{Au}$ film (13 nm) on glass and the 12 monolayer (ML) Au film on silicon match, which indicates a similar coverage of alkanethiols. For the $1.5 \mathrm{ML}$ film there is an additional feature $(285.8 \mathrm{eV})$ which is not characteristic of alkanethiols, and the intensity of the $(\mathrm{C}-\mathrm{H})$ and $(\mathrm{C}-\mathrm{C})$ resonances is greatly reduced. This indicates an incomplete alkanethiol layer. A similar result was observed for $6 \mathrm{ML}$ of Au. Therefore we conclude that a minimum of $12 \mathrm{ML}(3 \mathrm{~nm})$ of $\mathrm{Au}$ is required to obtain the full coverage.

Figures 3(a) and 3(b) show the polarization dependence of the $\mathrm{C} 1 s$ absorption spectra of alkanethiols. They were absorbed on $12 \mathrm{ML}$ of Au on silicon and on thick Au (13 nm) on glass. The polar angle between the light and the samples was varied and the data was normalized as before. A systematic but slight decrease in the height of the $\mathrm{C} 1 \mathrm{~s}$ edge for increasing scan number was observable $(\sim 12 \%$ over 8 scans). It is attributed to radiation damage of the alkanethiol films. As a first order correction, the levels of the carbon edge above the background (as measured by the intensity at $298 \mathrm{eV}$ ) were matched.
A comparison of the polarization dependence of the $\sigma^{*}$ peaks between the glass and Si samples reveals a similarity between the structures of the absorbed alkanethiol films. In both cases, the $\mathrm{C}-\mathrm{H} \sigma^{*}$ resonance is strongest at normal incidence $\left(0^{\circ}\right)$ and decreases as the polar angle is increased. Conversely, the $\mathrm{C}-\mathrm{C} \sigma^{*}$ resonance is weakest at normal incidence and increases as the polar angle is increased. This anisotropy indicates well-ordered films and is consistent with the widely used model in which the azimuthal distribution is random, but the molecules make a well-defined tilt angle with respect to the normal. After subtracting the background, the peak intensities versus polar angle are fit by curves of the form $\left(a+b \cos ^{2} \theta\right)$ or $\left(a+b \sin ^{2} \theta\right)$ [Figs. 3(c) and 3(d)]. For alkanethiols on glass we find $a=0.91, b=0.55$ for the $\mathrm{C}-\mathrm{C}$ $\sigma^{*}$ and $a=0.73, b=0.71$ for the $\mathrm{C}-\mathrm{H} \sigma^{*}$. Similarly, for alkanethiols on silicon we find $a=0.91, b=0.54$ for the $\mathrm{C}-\mathrm{C}$ $\sigma^{*}$ and $a=0.73, b=0.58$ for the $\mathrm{C}-\mathrm{H} \sigma^{*}$. The good agreement of the fit parameters between the two samples suggests a similar ordering and average tilt angle for alkanethiol films on the two substrates. If the carbon $1 s$ step edge is subtracted, the fit parameters can be used to determine the average tilt angle. ${ }^{22}$ Previous authors have used a building-block model which averages over the azimuthal distribution and the different bond angles within the molecule. ${ }^{20}$

For the stepped sample one might expect an azimuthal anisotropy. Because of the miscut angle away from the $\mathrm{Si}(111)$ plane, the (111) terraces make an angle of $\sim 3.5^{\circ}$ to the sample surface which should be reflected in the thiol overlayer. In Fig. 3(d), with the polarization vector perpendicular to the steps, this would appear as a shift in the maximum away from the sample normal. Conversely, if the polarization vector was parallel to the steps, there would be no shift. However, within experimental error this anisotropy is too small to distinguish.

No polarization dependent effects at all were observed for the alkanethiols absorbed on thinner gold films (6 ML and $1 \mathrm{ML}$ ) on silicon. Previous studies demonstrate that the completeness of an alkanethiol monolayer is crucial in determining its ordering. ${ }^{3,23}$ The absence of polarization dependence indicates a lack of ordering which accompanies an incomplete alkanethiol coverage. We propose that this lack of ordering may be due to defects and grain boundaries in the thin gold that allow the contamination of the surface in the ambient and in solution, and thus prevent the formation of complete, well-ordered monolayers. Conversely, silicon surfaces coated with $>3 \mathrm{~nm}$ of Au act very similarly to standard reference surfaces. For these thicker films defects do not penetrate to the substrate. The surface is effectively passivated and thus complete, well-ordered monolayers can be formed.

\section{B. DNA}

The $\mathrm{N} 1 s$ absorption spectra for DNA segments are shown in Fig. 4. We compare DNA adsorbed on glass and silicon, both coated with gold. All spectra are normalized to a clean gold film evaporated in situ on silicon. After the preedge backgrounds are normalized to one, the two curves overlay almost exactly, suggesting identical coverages of DNA on glass and silicon. The two preedge features are assigned to transitions from the $\mathrm{N} 1 s$ core level to unoccupied $\pi^{*}$ orbitals in the carbon-nitrogen double bonds of the DNA 


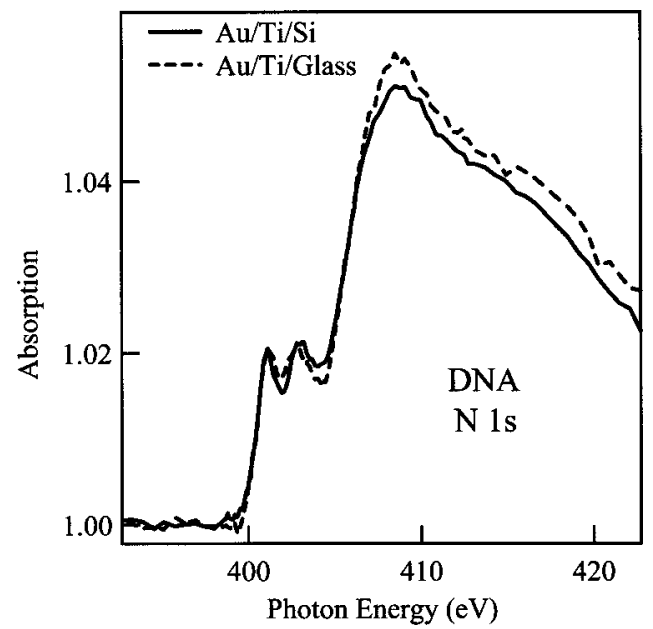

FIG. 4. N $1 s$ absorption of short, thiol-functionalized DNA segments attached to silicon and glass via a Au layer.

nucleotide bases. The splitting of the two $\pi^{*}$ orbitals $(\Delta E$ $=2.1 \mathrm{eV}$ ) and their position relative to the nitrogen edge agree with previously published results for double-stranded DNA. ${ }^{21}$ These features represent an average over the different nucleotide bases. The chemical shift of one $\pi^{*}$ orbital to higher energy may be partly due to the presence of exocyclic carbonyl groups in some of the bases. ${ }^{21}$ Higher energy resonances result from transitions to the $\sigma^{*}$ levels, giving rise to the broad, main peak.

Figure 5 shows $\mathrm{N} 1 s$ absorption spectra of DNA for different polar angles. The $\pi^{*}$ resonances are weakest at
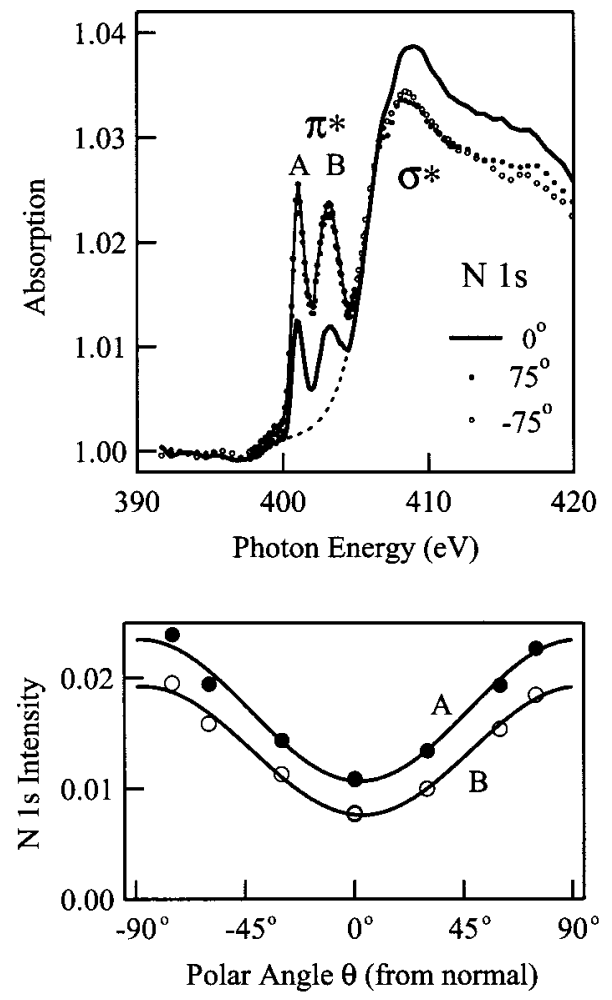

FIG. 5. Polarization-dependence of the N $1 s$ absorption edge of short DNA segments. The $\pi^{*}$ transitions $\mathrm{A}$ and $\mathrm{B}$ are stronger for a large angle of incidence $\vartheta$, where the component of $\mathbf{E}$ perpendicular to the surface is large. normal incidence and strongest at grazing incidence, while the $\sigma^{*}$ resonances are strongest at normal incidence and weakest at grazing incidence. Since the $\pi^{*}$ orbitals lie along the axis of the DNA, while the $\sigma^{*}$ orbitals are in the plane of the base pairs, the data imply that the axis of the DNA helix tends to stand away from the surface. Previous AFM studies have observed long DNA duplexes to lie with their helix axis parallel to surfaces. ${ }^{24}$ For short rod-like snippets, the interaction between adjacent DNA strands and the substrate to thiol bond angle prevent the molecules from lying flat. Recent studies have emphasized the importance of the thiolsubstrate bond in determining alkanethiol orientation. ${ }^{25}$ For DNA monolayers, where the packing is low, the thiol bond may be of particular importance in determining the orientation.

For determining the $\pi^{*}$ peak intensities a background was subtracted to remove the effects of the nearby $\sigma^{*}$ resonances (see the dashed line in Fig. 5). Plots of the peak intensity versus polar angle for the two $\pi^{*}$ resonances are fit with an $a+b \cos ^{2} \theta$ dependence yielding values of $a$ $=0.008, b=0.012$ and $a=0.011, b=0.013$ for peaks A and $\mathrm{B}$, respectively. Similarly to the alkanethiols this fit suggests an average tilt of the DNA axis of roughly $45^{\circ}$ away from the normal combined with a random azimuthal distribution. This result is consistent with earlier ellipsometry and AFM studies in which double-stranded DNA was absorbed onto Au surfaces using a similar method. ${ }^{26,27}$ The DNA monolayers are not as well ordered as the alkanethiol monolayers and thus the tilt angle is less well-defined. As a result fit values for the DNA varied from sample to sample. However, DNA monolayers on both glass and silicon samples exhibit the same orientation dependence within the experimental error. Since the $\mathrm{N} 1 s$ coverage is also the same we conclude that similar monolayers of DNA can be formed on $3 \mathrm{~nm}$ Au films on $\mathrm{Si}$ as on standard reference films on glass.

\section{SUMMARY AND OUTLOOK}

In summary we find that thiol derivatives of alkanes and DNA can be adsorbed on $\mathrm{Si}$, as long as silicon is functionalized by a gold layer at least $3 \mathrm{~nm}$ thick. For alkanethiols the maximum coverage is reached and the same preferential orientation is found as for the gold-coated glass surfaces used for this purpose previously. For short, double-stranded DNA segments (20 base pairs) we find an anisotropy with a preferred polar angle of roughly $45^{\circ}$ and a random azimuthal distribution, analogous to alkanethiols.

In future studies we aim to use these substrates to determine how biomolecules align and interact at stepped silicon templates. These interactions are particularly significant in template processes, such as the alignment of liquid crystals. ${ }^{6,7,12-15,28}$ Such processes are controlled by not only the molecular level structure, but also the nanometer-scale topography ${ }^{28,29}$ An example is the liquid crystal alignment observed on stepped silicon. ${ }^{29}$ Past studies have demonstrated that it is possible to exploit changes in liquid crystal alignment to detect the selective binding of biological species which have sizes that are comparable to the nano-scale patterning. ${ }^{7} 12-15,28$ 
Other interesting candidates for future study are metal clusters passivated by organic molecules and possibly functionalized by DNA snippets ${ }^{30}$ to bind to steps in regular arrays. Colloidal clusters of ferromagnetic materials ${ }^{31}$ might serve as storage elements in a single-particle-per-bit magnetic memory. In this case it is important to have these particles arranged in a regular pattern along straight step edges, such that they can be read out by an array of scanning probes. $^{32}$

\section{ACKNOWLEDGMENTS}

This work was supported by NSF under Award No. DMR-9815416 and No. DMR-0079983, and ONR under Award No. N000149910250. Part of it was conducted at the Synchrotron Radiation Center, University of WisconsinMadison, which is supported by the NSF under Award No. DMR-0084402.

${ }^{1}$ For an overview of molecular electronic devices, see Ann. N.Y. Acad. Sci. 852, 1 (1998).

${ }^{2}$ C. P. Collier, E. W. Wong, M. Belohradsky, F. M. Raymo, J. F. Stoddart, P. J. Kuekes, R. S. Williams, and J. R. Heath, Science 285, 391 (1999).

${ }^{3}$ C. D. Bain, E. B. Troughton, Y. T. Tao, J. Evall, G. M. Whitesides, and R. G. Nuzzo, J. Am. Chem. Soc. 111, 321 (1989).

${ }^{4}$ P. E. Laibinis, J. J. Hickman, M. S. Wrighton, and G. M. Whitesides, Science 245, 845 (1989).

${ }^{5}$ M. D. Porter, T. B. Bright, D. L. Allara, and C. E. Chidsey, J. Am. Chem. Soc. 109, 3559 (1987).

${ }^{6}$ V. K. Gupta and N. L. Abbott, Science 276, 1533 (1997).

${ }^{7}$ V. K. Gupta, J. J. Skaife, T. B. Dubrovsky, and N. L. Abbott, Science 279, 2077 (1998).

${ }^{8}$ T. M. Herne and M. J. Tarlov, J. Am. Chem. Soc. 119, 8916 (1997).

${ }^{9}$ T. Strother, W. Cai, X. Zhao, R. J. Hamers, and L. M. Smith, J. Am. Chem. Soc. 122, 105 (2000).

${ }^{10}$ M. A. Cooper, M. T. Fiorini, C. Abell, and D. H. Williams, Bioorg. Med. Chem. Lett. 8, 2609 (2000).

${ }^{11}$ M. Franco, P. F. Nealey, S. Campbell, A. I. Teixeira, and C. J. Murphy, J. Biomed. Mater. Res. 52, 261 (2000).
${ }^{12}$ J. J. Skaife and N. L. Abbott, Langmuir 16, 3529 (2000).

${ }^{13}$ J. J. Skaife and N. L. Abbott, Chem. Mater. 11, 612 (1999).

${ }^{14}$ J. J. Skaife, J. M. Brake, and N. L. Abbott, Langmuir (to be published).

${ }^{15}$ J. J. Skaife and N. L. Abbott, Langmuir (to be published).

${ }^{16}$ A. Kirakosian, J.-L. Lin, D. Y. Petrovykh, J. N. Crain, and F. J. Himpsel, J. Appl. Phys. 90, 3286 (2001), preceding paper.

${ }^{17}$ J. Viernow, J.-L. Lin, D. Y. Petrovykh, F. M. Leibsle, F. K. Men, and F. J. Himpsel, Appl. Phys. Lett. 72, 948 (1998); J.-L. Lin, D. Y. Petrovykh, J. Viernow, F. K. Men, D. J. Seo, and F. J. Himpsel, J. Appl. Phys. 84, 255 (1998).

${ }^{18}$ K. N. Altmann, J. N. Crain, R. Losio, A. Kirakosian, J.-L. Lin, D. Y. Petrovykh, and F. J. Himpsel, Phys. Rev. B 64, 035406 (2001).

${ }^{19}$ G. Hahner, M. Kinzler, C. Thummler, Ch. Woll, and M. Grunze, J. Vac. Sci. Technol. A 10, 2758 (1992).

${ }^{20}$ D. A. Outka, J. Stöhr, J. P. Rabe, and J. D. Swalen, J. Chem. Phys. 88, 4076 (1988); J. Stöhr, NEXAFS Spectroscopy (Springer-Verlag, Berlin, 1992).

${ }^{21}$ S. M. Kirtley, O. C. Mullins, J. Chen, J. van Elp, S. J. George, C. T. Chen, T. O'Halloran, and S. P. Cramer, Biochim. Biophys. Acta 1132, 249 (1992).

${ }^{22}$ Quantitative comparison with earlier results depends on the choice for the location and shape of the carbon $1 s$ edge.

${ }^{23}$ G. Hahner, C. Woll, M. Buck, and M. Grunze, Langmuir 9, 1955 (1993).

${ }^{24}$ Y. Maeda, T. Matsumoto, H. Tanaka, and T. Kawai, Jpn. J. Appl. Phys., Part 2 38, L1211 (1999).

${ }^{25}$ M. Zharnikov, S. Frey, H. Rong, Y.-J. Yang, K. Heister, M. Buck, and M. Grunze, Phys. Chem. Chem. Phys. 2, 3359 (2000).

${ }^{26}$ S. O. Kelley, J. K. Barton, N. M. Jackson, L. D. McPherson, A. B. Potter, E. M. Spain, M. J. Allen, and M. G. Hill, Langmuir 14, 6781 (1998).

${ }^{27}$ S. O. Kelley, J. K. Barton, N. M. Jackson, and M. G. Hill, Bioconjugate Chem. 8, 31 (1997).

${ }^{28}$ D. L. Patrick, V. J. Cee, M. D. Morse, and T. P. Beebe, J. Phys. Chem. B 103, 8328 (1999)

${ }^{29}$ E. H. Lay, A. Kirakosian, J.-L. Lin, D. Y. Petrovykh, J. N. Crain, R. R. Shah, N. L. Abbott, and F. J. Himpsel, Langmuir 16, 6731 (2000).

${ }^{30}$ C. A. Mirkin, MRS Bull. 25, 43 (2000).

${ }^{31}$ S. Sun, C. B. Murray, D. Weller, L. Folks, and A. Moser, Science 287, 1989 (2000).

${ }^{32}$ G. Binnig, M. Despont, U. Drechsler, W. Häberle, M. Lutwyche, P. Vettiger, H. J. Mamin, B. W. Chui, and T. W. Kenny, Appl. Phys. Lett. 74, 1329 (1999). 